\section{A storm in a teacup: older adults' low prevalence of COVID-19 vaccine side-effects and their link with vaccination anxiety}

The widespread COVID-19 pandemic led to unprecedentedly rapid vaccination development (Graham, 2020). One topic less addressed is vaccine side-effects and their link with anxiety, a condition detrimental to older adults (Bodner et al., 2021). General anxiety was found in $2.4 \%$ of those receiving the Pfizer vaccination (Kadali et al., 2021). Based on the Johnson \& Johnson, Janssen vaccination findings, Hause et al. (2021) suggested that common side-effects typical of anxiety, for example, nausea, were driven by anxiety. As psychological factors may impact the immune system (Madison et al., 2021), anxiety may even link with side-effects atypical of anxiety (e.g., swollen-lymph-nodes). In addition to general anxiety (Kadali et al., 2021), research showed that vaccination itself may trigger anxiety, for example, its rapid development or an irrational misinformed perception of it being more dangerous than COVID-19 (Bodner et al., 2021). Thus, we examined in older adults the linking of vaccination anxiety with vaccination side-effects (both typical and atypical of anxiety), whilst controlling for general anxiety.

A representative Israeli sample of vaccinated community older adults participated in this study $(N=939$, mean age $68.9 \pm 3.43$, range 65-85; $59.9 \%$ females, $47.2 \%$ with academic education; $75.5 \%$ married/living with partner). The study was conducted between January $25^{\text {th }}$ and February $4^{\text {th }}, 2021$; on January 25 cumulative COVID-19 vaccination doses administered per 100 people were 46.73, and on February 4, 62.72. Participants provided informed consent to procedures approved by authors' university institutional review board and responded 28.15 \pm 9.47 days after the first of two Pfizer BNT162b2 mRNA COVID-19 vaccinations.

Questionnaire were disseminated via a polling company. Participants rated (1-not suffering at all to 5-suffering very severely) each vaccination sideeffect (based on the FDA ${ }^{1}$ and Israeli Ministry of Health $^{2}$ ), see Table 1. A single item asked to "please rate their vaccination anxiety" (1-not-at-all to 5very-much). We also assessed general anxiety levels

\footnotetext{
${ }^{1}$ https://www.fda.gov/media/144414/download ${ }^{2}$ https://en.globes.co.il/en/article-covid-vaccine-side-effects-in-israel-matchtrials- 1001359338
}

(GAD-7, Spitzer et al., 2006, $\alpha=0.94$ ). Additionally, participants were asked to subjectively rate their general health (ranging from 1-very bad to 5-very good, Idler and Benyamini, 1997).

Side-effects were rare (see Table 1). The average side-effect score was unrelated to number of days from vaccination, higher in females (1.26 vs. 1.18 , $t=3.81, \quad p<0.0001)$, decreased with age $(r=-0.10, p<0.0001)$, negatively linked with subjective health $(r=-0.19, p<0.0001)$, positively associated with GAD $(r=0.23, p<0.0001)$, and vaccination anxiety $(r=0.399, p<0.0001)$. Neither GAD nor vaccination anxiety linked with "days since vaccination" ( $p$ 's $>0.63$ ).

Correlations of anxiety measures with side-effects both typical (see Table 1, depicted by ${ }^{2}$ ) and atypical of anxiety were respectively similar (GAD, $r=0.209, p=0.0001$ vs. $r=0.224, p=0.0001$; vaccination anxiety, $r=0.341, p=0.0001$, vs. $r=0.39$, $p=0.0001)$. Limiting typical side-effects to nausea, chills, headaches, and dizziness yielded the same results. Critically, partial correlations show that vaccination anxiety maintained its link with sideeffects even after controlling for GAD and subjective health $(r=0.36, p=0.001)$.

These results rejoin previous results attesting to vaccination safety and to side-effects decreasing with age (Polack et al., 2020). Vaccination anxiety (Bodner et al., 2021) did not dimmish with time since vaccination, suggesting that such anxiety is less rational. Vaccination side-effects, both typical and atypical of anxiety, similarly linked with anxiety, indicating that rather being driven by overlapping symptoms (Hause et al., 2021), anxiety perhaps impacts the immune system (Madison et al., 2021). Although vaccination anxiety also stems from irrational misinformation (Berry et al., 2021), results suggest that it may be important to one's physical health.

Alongside this study's strengths (e.g., a large representative sample, measuring both typical and atypical side-effects and their link with vaccination anxiety, whilst controlling for both subjective health and GAD), limitations are noted. First, we focused on a specific age group and a single vaccination. Moreover, we used a single vaccination-anxiety item which may not as reliable or understood as the longer index (Bodner et al., 2021). Furthermore, although slightly mitigated by the above partial correlations, future research should control for additional factors that might impact anxiety level, for example, therapy, psychiatric, or medical history. Finally, causality could not be discerned 
Table 1. Distribution of side-effect severity following the Pfizer BNT162b2 mRNA COVID-19 vaccine $(n=939)$

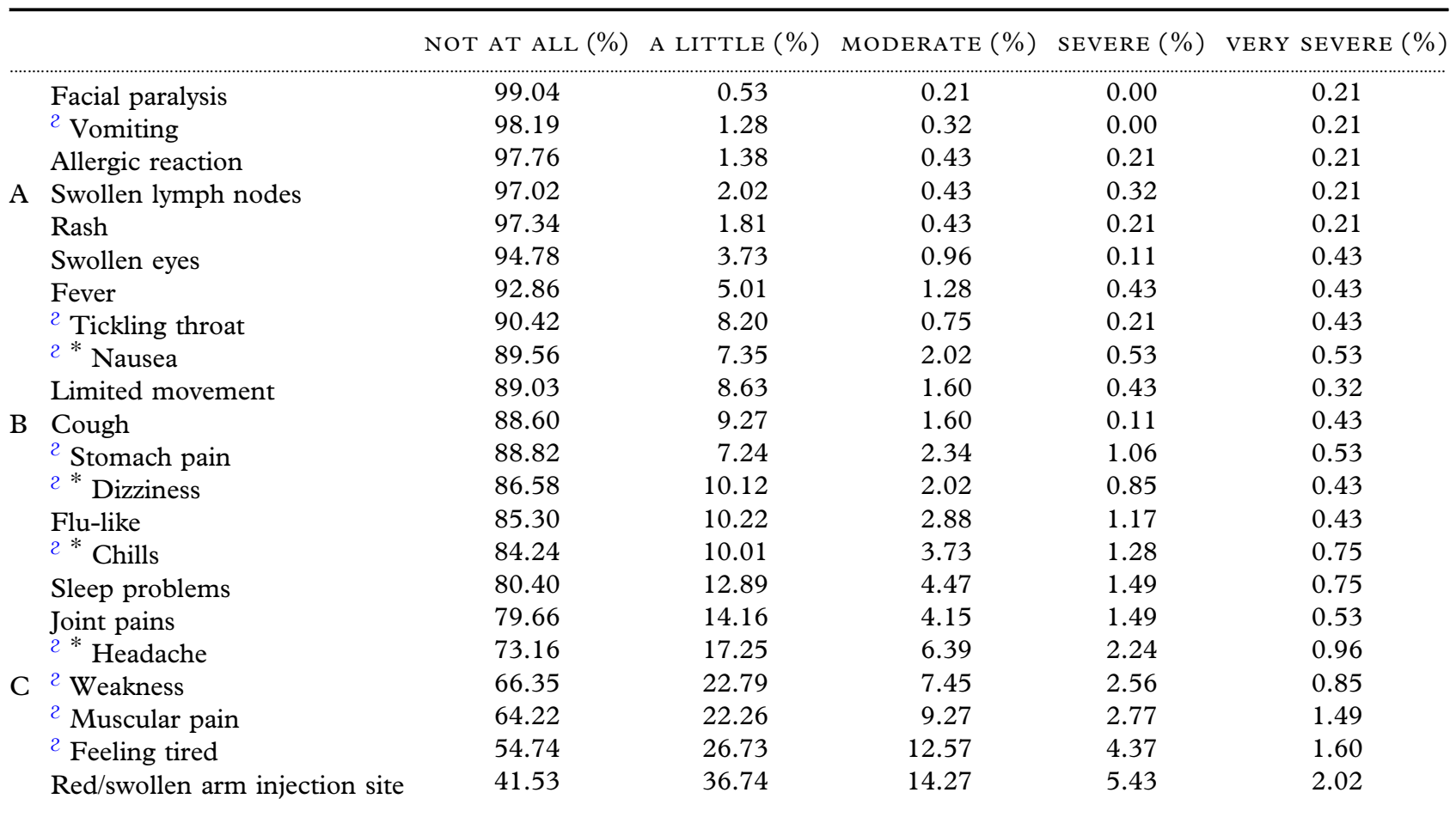

${ }^{2}$ Liberal depiction of side-effects that may be typical of anxiety; * Conservative depiction of side-effects that may be typical of anxiety

(side-effects driving anxiety or vice-a-versa) in this cross-sectional study.

Yet given the global scale of vaccination programs, the strong link between vaccination anxiety and vaccination side-effects is important both for COVID-19 and perhaps for future pandemics. Data showing infrequent side-effects alongside a relatively robust psychosomatic component may encourage vaccination (Berry et al., 2021). Results can aid in identifying persons with anxiety; in turn suitable and effective help to ameliorate this anxiety may be offered, which may possibly alleviate side-effects. Following results, vaccination anxiety (Bodner et al., 2021) like COVID-19 anxiety (Bergman et al., 2020) may benefit from considering strategic health policies that address realistic dangers of vaccination vis-à-vis COVID-19 itself (Berry et al., 2021).

\section{Acknowledgements}

We thank the research authority at Ariel University for their continued support in basic research.

\section{Conflict of interest}

The authors have no conflicts of interest to declare.

\section{Author contributions}

Dr Ben-Ezra obtained an internal grant from Ariel University. Drs. Greenblatt-Kimron, Palgi, and
Ben-Ezra initiated this project. Drs. Palgi, Greenblatt-Kimron, and Ben-Ezra compiled a questionnaire and organized data collection. Drs. Hoffman and Ben-Ezra conceived the idea for this study and together with Dr Goodwin analyzed the data. Drs. Hoffman and Ben-Ezra, drafted the manuscript. Drs Hoffman, Ben-Ezra, and Goodwin wrote the first manuscript version. All authors wrote, rewrote, and further edited the manuscript. All authors had full access to all of the data in the study. All authors take responsibility for the integrity of the data and the accuracy. All authors provided substantially to the drafting and revising of this manuscript.

\section{Sponsor's role}

The study was funded by an internal grant awarded to Dr Menachem Ben-Ezra from Ariel University. The sponsor had no role in the study design or interpretation of the data.

\section{References}

Bergman, Y. S., Cohen-Fridel, S., Shrira, A., Bodner, E. and Palgi, Y. (2020). COVID-19 health worries and anxiety symptoms among older adults: the moderating role of ageism. International Psychogeriatrics, 32, 1371-1375.

Berry, S. D. et al. (2021). Lessons learned from frontline skilled nursing facility staff regarding COVID-19 vaccine 
hesitancy. Fournal of the American Geriatrics Society. https:// doi.org/10.1111/jgs. 17136

Bodner, E., Bergman, Y. S., Ben-David, B. and Palgi, Y. (2021). Vaccination Anxiety when Vaccinations are Available: The Role of Existential Concerns. Stress and Health. https://doi.org/10.1002/smi.3079

Hause, A. M. et al. (2021). Anxiety-related adverse event clusters after Janssen COVID-19 vaccination-five US mass vaccination sites, April 2021.

Graham, B. S. (2020). Rapid COVID-19 vaccine development. Science, 368, 945-946. https://doi.org/10. 1126/science.abb8923

Kadali, R. A., Janagama, R., Peruru, S. and Malayala, S. V. (2021). Side effects of BNT162b2 mRNA COVID-19 vaccine: a randomized, cross-sectional study with detailed self-reported symptoms from healthcare workers. International Fournal of Infectious Diseases, 106, 376-381.

Idler, E. L. and Benyamini, Y. (1997). Self-rated health and mortality: a review of twenty-seven community studies. Fournal of Health and Social Behavior, 38, 21-37.

Madison, A. A., Shrout, M. R., Renna, M. E. and Kiecolt-Glaser, J. K. (2021). Psychological and behavioral predictors of vaccine efficacy: considerations for COVID-19. Perspectives on Psychological Science, 16, 191-203.
Polack, F. P. et al. (2020). Safety and efficacy of the BNT162b2 mRNA Covid-19 vaccine. New England Fournal of Medicine, 383, 2603-2615.https://doi.org/10.1056/ NEJMoa2034577

Spitzer, R. L., Kroenke, K., Williams, J. B. and Löwe, B. (2006). A brief measure for assessing generalized anxiety disorder: the GAD-7. Archives of Internal Medicine, 166, 1092-1097. https://doi.org/10. 1001/archinte.166.10.1092

YaAkov Hoffman, ${ }^{1}$ (i) Yuval Palgi, ${ }^{2}$ Robin Goodwin, ${ }^{3}$ Menachem Ben-Ezra ${ }^{4}$ and LEE GreEnBlatt-KImRON ${ }^{4}$

${ }^{1}$ Interdisciplinary Department of Social Sciences, Bar-Ilan University, Ramat-Gan, Israel

${ }^{2}$ Department of Gerontology, University of Haifa, Haifa, Israel

${ }^{3}$ Department of Psychology, Warwick University, Coventry, UK

${ }^{4}$ School of Social Work, Ariel University, Ariel 40700, Israel

Correspondence should be addressed to: Dr. Yaakov S.G. Hoffman, Email: hoffmay@biu.ac.il; hoffmay@gmail. com. 